\title{
The Chemistry of Paper in Paper Spray Ionization Mass Spectrometry
}

\author{
Wejdan T. Alsaggaf ${ }^{1,2}$ \\ ${ }^{1}$ Department of Chemistry, King Abdulaziz University, P.O. Box 42906, Jeddah 21551, Kingdom of Saudi Arabia \\ ${ }^{2}$ Department of Chemistry, University of British Columbia, British Columbia, V1V1V7, Canada \\ Correspondence: Wejdan T. Alsaggaf, Assistant professor, Chemistry department, Room 4 Science College, King \\ Abdulaziz University, P.O. Box 42906, Jeddah 21551, Kingdom of Saudi Arabia. E-mail: wejdantalsaggaf@gmail.com
}

Received: August 27, 2019 Accepted: October 9, 2019 Online Published: October 10, 2019

doi:10.5539/ijc.v12n1p16

URL: https://doi.org/10.5539/ijc.v12n1p16

\begin{abstract}
There are many rapidly evolving technologies that use simple papers as a surface for chemical reactions and detection of molecules. One example of this application is the use of paper as a surface for ionization and introduction of compounds into mass spectrometry. The current work was designed to investigate mechanisms of interaction between the paper and analytes using mass spectrometry as the detection instrument. Standard compounds with different sizes were used in this study. Factors investigated include the effects of the paper geometry $(5 \mathrm{~mm})$ and position $\left(\mathrm{tp}=90^{\circ}\right)$ were found to be optimal. The role of atmospheric water and the effects of paper porosity were affected the signals of the tested compounds. Investigations of the interaction of standard solutions with the paper surface indicated that atmospheric water is required for ionization. Up to three water molecules were detected in association with the analytes indicating that the presence of some water is required. In addition, this study showed the potential of silicon dioxide nanoparticles for eluting the standards compound from the paper surface. The impact of trace elements in the commercial papers and the interactions between the paper cellulose with different classes of analytes was investigated.
\end{abstract}

Keywords: paper spray ionization, water clusters, nanoparticles

\section{Introduction}

Several recent projects have developed new analytical instruments and tools using paper as a substrate for chemical reactions and analyte detection. Among the most interesting is a new paper-based device for detection strain specific detection of Ebola. The expansion of possible uses for paper-based technologies necessitates studies to better understand the chemistry of the paper substrate and the interactions between paper and analytes. Paper Spray Ionization (PSI) is a relatively new ambient ionization technique for mass spectrometry (Kim et al., 2018; Yu et al., 2018; Liu et al., 2017; Bills, \&Manicke, 2016; Carvalho et al., 2016; Liu et al., 2010; Wang, Liu, Cooks, \& Ouyang, 2010) that allows for the direct detection of analytes from the paper surface.

In Paper Spray Ionization (PSI) a triangular shaped filter paper substrate is used to collect a sample and then analyte ions are produced by applying a high electric field and a flush of solvent (Liu et al., 2010; Wang et al., 2010; Espy, Muliadi, quyang, Cooks, 2012). Typically, samples are preloaded on the paper substrate in a solution by injecting sample on substrate using syringe.Ions are produced by applying a small amount of solvent and electrical potential to the paper substrate (Liu et al., 2010; Espy et al., 2012). In PSI, an electric field is generated when applied to the high electric potential between the paper substrate and a mass spectrometry inlet (Liu et al., 2010). It was hypothesized that this phenomenon is similar to electrospray ionization (ESI), where charged droplets are formed when the electric field becomes higher than the surface tension of the liquid at the paper tip (Liu et al., 2010; Espy et al., 2012). Charged droplets were produced from a Taylor cone that forms at the tip of the paper. The charged droplets undergo a series of desolvation processes leading to the formation of the analyte ions (Espy et al., 2012). However, the consistency of the edges of the paper can affect the analysis with 5 different Taylor cones with different trajectories observed (Espy et al., 2012). Some electron-transfer species such as radical cations or anions, and typical Bronsted acid-base species and/or salt adducts have also been observed (Liu et al., 2010; Espy et al., 2012). In addition, the solvent used to dissolve the sample and carry the current affected the ionization efficiency in some systems with non-polar solvents applied to the paper effectively ionizing drugs, peptides, nucleotides, and phospholipids in the absence of aqueous solvents (Page, Kelly, Tang, \& Smith 2007; Li, Wang, Quyang, \& Cooks, 2011). However, the overall objective of this study was to determine the chemistry of the paper and identifying any possible chemical interferences introduced by paper substrates.

To achieve the overall objective of these studies, several specific objectives were investigated. In preliminary studies, the 
physical properties of the paper substrate were optimized including the type of paper and the paper geometry, position and porosity. In the present work, we hypothesized that ionization in the presence of non-polar solvents was a result of atmospheric water molecules that adhere to the paper surface. To determine whether the paper introduced additional matrix effects to the analysis, we analyzed the ions driven off the surface in the absence of samples. To investigate the effects of the paper pore sizes on the analysis, we introduced a film of silica nanoparticles that reduced pore size and serve as a proton donor to boost the ionization efficiency. Finally, we the potential investigated the mechanisms of separation of analytes across the paper surface as a technique for analysis of complex sample matrices. Together these data demonstrate the utility of the paper surfaces for many different types of analyses and indicate some important factors for consideration.

\section{Experimental}

\subsection{Chemicals and Materials}

Analytical standards were: 17 $\alpha$-hydroxyprogesterone (C21H30O3, 95\% purity; Sigma), 3, 3, 5-triiodo-L-thyronine (T3; C15H12I3NO4, 95\% purity; Sigma), astaxanthin (C40H52O4, all-trans; 97\% purity; Sigma), $\beta$-carotene (C40H56, Type $1,95 \%$ purity), $\beta$-ionone (C13H20O, $\geq 95 \%$ purity, Fluka), caffeine (C8H10N4O2, 99\% purity; Sigma /Fluka), crocin (C44H64O24, microscopy grade), cortisone (C21H28O5, 98\% purity; Sigma), estriol (C18H24O3, 98\% purity; Sigma-Aldrich), estrone (C18H22O2, 99\% purity; Sigma), hydrocortisone (C21H30O5, 98\% purity; Sigma), isophorone (C9H14O, 97\% purity, Sigma), kaempferol (C15H10O6, High performance liquid chromatography (HPLC) Grade; 97\% purity), L-proline (C5H9NO2, $\geq 99.5 \%$ purity, Sigma), quercetin (C15H10O7, Anhydrous, Q4951, Sigma), safranal (C10H14O, $\geq 88 \%$ purity, Sigma), thyroxin (T4; C15H11I4NO4, Cell Culture Grade; Sigma), and zeaxanthin (C40H56O2, 95\% purity, Sigma-Aldrich, St. Louis, MO). Filter papers compared were standard filter papers (Whatman Grade 1 (G1), Whatman Grade 4 (G4), and Whatman Grade 5 (G5); Fisher, Mississauga ON), glass microfiber filter (GF; Whatman GF/A; Fisherbrand G6), and silicon-treated phase separator (1PS; Whatman 1PS phase separator) (Table 1). Polar and non-polar solvents were: acetone (HPLC Grade; Fisher), acetonitrile (ACS/HPLC Grade; Fisher), dichloromethane (HPLC Grade; Fisher), hexane (Optima ACS; Fisher), methanol (Optima Liquid Chromatography-Mass Spectrometry (LC-MS); Fisher), pentane (HPLC Grade; Fisher) and toluene (HPLC Grade; Fisher). Silicon nanoparticles were $<50 \mathrm{~nm}$ particle size (Silicon dioxide, alumina doped, 99.99\% purity in solution; Sigma-Aldrich).

Table 1. Characteristic of six types of paper

\begin{tabular}{lcccc}
\hline Types & Porosity & Flow rate & $\begin{array}{c}\text { Particle } \\
\text { retention }(\mu \mathrm{m})\end{array}$ & Uses \\
\hline $\begin{array}{l}\text { Whatman } \\
\text { Grade } \mathbf{1}\end{array}$ & Medium & Medium & 11 & $\begin{array}{l}\text { Clarifying liquids, gas filtration, } \\
\text { analysis of air and soil pollution }\end{array}$ \\
\hline $\begin{array}{l}\text { Whatman } \\
\text { Grade 4 }\end{array}$ & Coarse & Fast & $20-25$ & $\begin{array}{l}\text { For rapid filtering with excellent } \\
\text { retention of coarse particles and } \\
\text { gelatinous precipitates }\end{array}$ \\
$\begin{array}{l}\text { Whatman } \\
\text { Grade 5 }\end{array}$ & Fine & Slow & 2.5 & Clarification of cloudy solutions \\
\hline $\begin{array}{l}\text { Whatman } \\
\text { GF/A }\end{array}$ & Fine & Fast & 1.6 & $\begin{array}{l}\text { For filtration of precipitated } \\
\text { protein, cell and cell debris. }\end{array}$ \\
$\begin{array}{l}\text { Fisherbrand } \\
\text { G6 }\end{array}$ & Fine & Fast & $3 \mu \mathrm{m}$ & $\begin{array}{l}\text { Chemically and biologically } \\
\text { inert, with no organic binders }\end{array}$ \\
$\begin{array}{l}\text { Whatman } \\
\text { lPS Phase } \\
\text { Separator }\end{array}$ & Medium & Medium & 11 & Rapid phase separator. \\
\hline
\end{tabular}




\subsection{Analytical Standards}

Standard stock solutions of each compound (20 mg of standard in $20 \mathrm{mLs}$ of solvent) were used to prepare diluted standards with final concentration of $0.51,5,10,30,50,150,200$, and $250 \mu \mathrm{g} / \mathrm{mL}$ in dark vials with a total volume of 3 mLs. Polar compounds such as 17 $\alpha$-hydroxyprogesterone, caffeine, cortisone, crocin, estriol, estrone, hydrocortisone, kaempferol and zeaxanthin were dissolved in 100\% methanol. Thyroid hormones (T3 \& T4) were dissolved in methanol with few drops $(200 \mu \mathrm{L})$ of ammonia solution $(\mathrm{NH} 4 \mathrm{OH})$. Moderate and non-polar compounds, including astaxanthin and $\beta$-carotene were dissolved in $100 \%$ dichloromethane. Dilutions of astaxanthin and $\beta$-carotene were made in $100 \%$ acetone while all other standards were diluted in $100 \%$ methanol. Standard stock solutions and dilutions were stored at $4{ }^{\circ} \mathrm{C}$ in dark vials for a maximum of 1 week and then replaced with new standards.

\subsection{Custom Instrumentation}

To investigate the chemistry of paper, a custom-built apparatus was constructed from simple materials that allowed for positioning of the paper and application of current (Figure 1). The PSI platform consisted of a non-conductive plastic housing supporting a metal clip (Figure 1B) that could be positioned into the mass spectrometer via the side portal (Figure 1A). The metal clip was used to hold the paper at precise distance and angle (Figure 1C). The eluent solvent was fed directly onto the paper via the Hamilton syringe pump and custom tubing (Figures 1B and D). Paper was cut into a triangle with dimensions: height $10 \mathrm{~mm}$ and base 5 according to standard shape that made of plastic (Figure 1B). A small amount of analyte $(3 \mu \mathrm{L})$ was loaded onto each of the papers directly. A variety of eluents including acetone, acetonitrile, hexane, methanol, pentane, and toluene were applied to the paper with a constant flow via the standard infusion syringe (Hamilton Syringe Pump; Hamilton Co., Reno, NV) at $10 \mu \mathrm{L} / \mathrm{min}$ (Figure 1D). After preliminary experiments, $100 \%$ methanol (10 $\mu \mathrm{L} / \mathrm{min}$ ) was selected as the most efficient solvent for $\beta$-ionone, cortisone, hydrocortisone, isophorone, querecetin, safranal and 17 $\alpha$-hydroxyprogesterone. Analysis of T3 and T4 were done with a mixture of acetonitrile and acetone (1:1). Esteriol and esterone eluted best with the mixture of acetonitrile and methanol (1:1). Astaxanthin, $\beta$-carotene and kaempferol were analyzed with $100 \%$ acetone as the eluent. Current was applied to the apparatus using the Multi Mode Ionization Source electrical outlet of the mass spectrometer (Waters Quattro Premier MS/MS; Waters, Millford MA) using MassLynx software to control voltage through the tune page over the range $1.5-4.5 \mathrm{KV}$ (Table 2). In preliminary experiments, the voltages applied to the paper surface were optimized as follows: astaxanthin $-3.0 \mathrm{KV}$, $\beta$-carotene -3.30 $\mathrm{KV}$, caffeine - $3.50 \mathrm{KV}$, cortisone - $3.50 \mathrm{KV}$, estriol $-3.23 \mathrm{KV}$, estrone - $3.31 \mathrm{KV}$, hydrocortisone - $3.63 \mathrm{KV}$, $17 \alpha$-hydroxyprogesterone $-3.66 \mathrm{KV}, \mathrm{T} 3-3.5 \mathrm{KV}$, and T4 - $3 \mathrm{KV}$.

Table 2. Mass spectrometry optimized parameters in positive ion mode and the expected ions for each compound

\begin{tabular}{|c|c|c|c|c|c|}
\hline \multirow[b]{2}{*}{ Compound } & \multicolumn{2}{|c|}{ Triple quadrupole } & \multicolumn{2}{|l|}{ TOF } & \multirow[b]{2}{*}{ Predominant } \\
\hline & $\begin{array}{l}\text { Applied } \\
\text { voltage } \\
(\mathrm{KV})\end{array}$ & $\begin{array}{l}\text { Cone } \\
\text { voltage } \\
\text { (V) }\end{array}$ & $\begin{array}{l}\text { Applied } \\
\text { voltage } \\
(\mathrm{KV})\end{array}$ & $\begin{array}{l}\text { Cone } \\
\text { voltage } \\
\text { (V) }\end{array}$ & \\
\hline $\begin{array}{l}17 \alpha- \\
\text { hydroxyprogesterone }\end{array}$ & 3.66 & 35 & 3.50 & 35 & {$[\mathrm{M}+\mathrm{H}]^{+}$} \\
\hline $\mathrm{T}_{3}$ & 3.5 & 41 & 3.50 & 35 & {$[\mathrm{M}+\mathrm{H}]^{+}$} \\
\hline $\mathrm{T}_{4}$ & 3 & 45 & 3.50 & 35 & {$[\mathrm{M}+\mathrm{H}]^{+}$} \\
\hline Astaxanthin & 3 & 35 & 3.30 & 35 & {$[\mathrm{M}+\mathrm{H}]^{+}$} \\
\hline$\beta$-Carotene & 3.30 & 35 & 3.50 & 35 & {$[\mathrm{M}+\mathrm{H}]^{+}$} \\
\hline Cortisone & 3.5 & 45 & 3.70 & 33 & {$[\mathrm{M}+\mathrm{H}]^{+}$} \\
\hline Hydrocortisone & 3.63 & 45 & 3.50 & 35 & {$[\mathrm{M}+\mathrm{H}]^{+}$} \\
\hline Estriol & 3.23 & 35 & 3 & 30 & {$[\mathrm{M}+\mathrm{H}]^{+}$} \\
\hline Estrone & 3.31 & 31 & 3.30 & 35 & {$[\mathrm{M}+\mathrm{H}]^{+}$} \\
\hline Caffeine & 3.50 & 31 & 3.50 & 35 & {$[\mathrm{M}+\mathrm{H}]^{+}$} \\
\hline Crocin & - & - & 3.50 & 35 & {$[\mathrm{M}+\mathrm{H}]^{+}$} \\
\hline Kaempferol & - & - & 3.50 & 35 & {$[\mathrm{M}+\mathrm{H}]^{+}$} \\
\hline Zeaxanthin & - & - & 3.50 & 35 & {$[\mathrm{M}+\mathrm{H}]^{+}$} \\
\hline
\end{tabular}



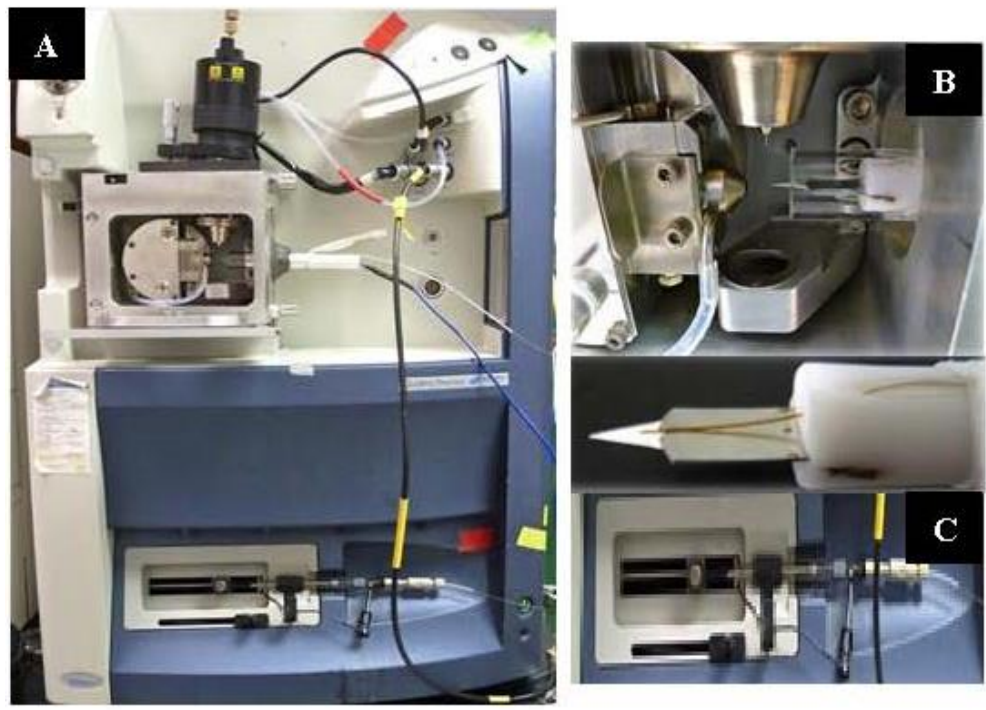

Figure 1. Triple quadrupole mass spectrometry in the left side, and (A) lab-made paper spray ionization apparatus where the paper should adjacent to MS inlet cone. (B) Solvent will pass through the paper by using capillary tube. (C) Infused injection method

\subsection{Mass Spectrometers}

Two different mass spectrometers were used for the PSI experiments to characterize the interactions between analytes and the paper substrate. A tandem MS/MS system (Waters Quattro Premier ${ }^{\mathrm{TM}}$ LCT MS/MS was used as a single quadrupole instrument to detect parent ions only. The second mass spectrometer was a time of flight MS (LCT Premier ${ }^{\mathrm{TM}}$ TOF/MS (Waters). Cone voltage and other tuning parameters were optimized by direct infusion of the $10 \mu \mathrm{g} / \mathrm{mL}$ standard solution (Hamilton Syringe Pump). Voltage applied to the custom paper support was optimized by analysis of replicate applications of standards to the paper surface $(0.5-250 \mu \mathrm{g} / \mathrm{mL}$ ) with varying voltage (Table 2). The MS scanned from 80 to $1000 \mathrm{Da}$ with a span of $0.2 \mathrm{Da}$, a dwell time of $0.5 \mathrm{~s}$, and a scan frequency of $1 \mathrm{~s}$. Three ionization techniques were utilized including the custom PSI apparatus, electrospray ionization (ESI), and electrospray chemical ionization (ESCI). Nitrogen gas (NitroFlow nitrogen generator; Parker Balston, Haverfill, MA) was supplied to the ESI and ESCI interface at 101 psi with desolvation at $500 \mathrm{~L} / \mathrm{h}$, with a corresponding N2 gas flow of $800 \mathrm{~L} / \mathrm{h}$. The source temperature was set to $120^{\circ} \mathrm{C}$, and the cone gas flow was $50 \mathrm{~L} / \mathrm{h}$ with ESI and ESCI. The standard solutions and/or solvents were introduced by constant direct infusion by syringe infusion $(0.030 \mathrm{~mL} / \mathrm{min})$ into an electrospray ionization (ESI) and electrospray chemical ionization (ESCI) sources, while it was $10 \mu \mathrm{L} / \mathrm{min}$ into a PSI. No temperature or nebulizer gas were utilized with PSI. In preliminary studies, cone voltages were optimized over the range from $10-50 \mathrm{~V}$ with maximum response values at: astaxanthin $-35 \mathrm{~V}, \beta$-carotene $-35 \mathrm{~V}$, caffeine $-35 \mathrm{~V}$, cortisone $-45 \mathrm{~V}$, estriol $-35 \mathrm{~V}$, estrone - $31 \mathrm{~V}$, Hydrocortisone -45 $\mathrm{V}, 17 \alpha$-hydroxyprogesterone $-35 \mathrm{~V}, \mathrm{~T} 3-41 \mathrm{~V}, \mathrm{~T} 4-45 \mathrm{~V}$ were found.

\subsection{Properties of Paper}

Preliminary experiments optimized the size, shape and orientation of the paper for signal intensity. A plastic template was used to cut the paper into standard shape and size with dimensions of $5 \mathrm{~mm}$ x $10 \mathrm{~mm}$ and with a $90^{\circ}$ angle tip. Orientation of the tip was found to be optimal at $90^{\circ}$ to the inlet cone. After preliminary experiments, Whatman Grade 1 paper was selected as the most efficient ionization support and used for further studies. To test the effects of atmospheric conditions such as humidity, Whatman Grade 1 paper was compared under ambient conditions and after being dried at $85^{\circ} \mathrm{C}$ for 24 hours (Isotemp Oven, Fisher).

\subsection{Determination of Analytical Performance}

Analytical performance was determined by IUPAC standard protocols for dynamic linear range, limit of detection (LOD) and limit of quantification (LOQ), (J. Miller, \& N. Miller, 1994).

\subsection{Paper as a Separation Surface}

To determine the capacity of the paper as a separation surface for complex samples, 4 sizes of Whatman Grade 1 paper $(0.5,2,4$, and $5 \mathrm{~cm}$ long $)$ were inoculated with a mixture of astaxanthin, crocin or zeaxanthin A $(10 \mu \mathrm{g} / \mathrm{mL}$ standard solution; $5-12 \mu \mathrm{L}$ applied to the paper) a voltage of $3.5 \mathrm{KV}$ was applied to the paper with a constant flow of $100 \%$ methanol at $10 \mu \mathrm{L} / \mathrm{min}$. Movement of the coloured pigments across the paper was visually observed and, when zeaxanthin 
neared the tip of the paper, voltage was removed and the distance migrated was measured with a standard ruler. These results were compared to traditional paper chromatography using Whatman Grade $1(5 \mathrm{~cm} \mathrm{long}$ ) with 100\% methanol eluent and the same standard solution and thin layer chromatography of the same standard solution on an aluminum-backed standard silica plate ( $200 \mathrm{~mm}$ x $200 \mathrm{~mm}$, cut to $5 \mathrm{~cm}$ lengths; VWR, Mississauga, ON) eluted with $100 \%$ methanol. The same standard ruler was used to measure the migration distances of the standards and relative retention factors (Rf) were calculated.

\subsection{Paper Matrix Effects}

To determine whether the paper surface contributes to the sample matrix complexity, Whatman Grade 1 paper triangles were positioned on the custom PSI apparatus in the Time of Flight mass spectrometer and $100 \%$ methanol was applied by direct infusion onto the paper surface via a Waters 1525 Binary syringe pump at $10 \mu \mathrm{L} / \mathrm{min}$ for 25 minutes. A total ion chromatogram (TIC) was generated for each paper exported to Microsoft Excel for untargeted statistical analysis to quantify the total number of signals produced by the paper substrate without addition any sample.

\subsection{Silica Nanoparticles}

Standard Whatman Grade 1 paper triangles were immersed in the silica dioxide nanoparticle solution for $0.25,24,72$, or 96 hours. After immersion, papers were dried under low flow of N2 gas for 1-2 mins prior to use in the PSI.

\subsection{Paper Substrate Imaging}

The images of paper substrates were obtained using the Tescan Mira3 XMU Field Emission Scanning Electron microscope (SEM) with variable pressure (up to $150 \mathrm{~Pa}$ ) in the UBC Okanagan Facility for Scanning-Electron Microscopy (Tescan Mira3 XMU, Czech Republic). Element analysis was performed with Oxford AZtec X-Max EDS system (Czech Republic) with $80 \mathrm{~mm}^{2}$ silicon drift detection.

\section{Results}

The studies were initiated to investigate the chemistry of paper substrates in chemical analyses. The objectives included investigation of the physical parameters of the paper and also the potential chemical interferences. Geometric parameters such as paper tip position (tp) and distance from paper tip to MS inlet (tip distance $=$ td) were optimized. A td of $5 \mathrm{~mm}$ was found to be optimal with shorter distances reducing the detection efficiency. The position of the tip (tp) was compared from $60^{\circ}-140^{\circ}$ rotation and the optimal position was tp $=90^{\circ}$ (Figure 2). Visual observation of the custom apparatus detected an electrical flash in the source that might be a corona discharge when a short distance $(<5 \mathrm{~mm})$ between paper tip and MS inlet cone (td) was investigated. The applied voltage and RF values were also standardized (Table 2). The five types of paper were compared to determine the relative efficiency and porosity influences of different substrates including Whatman glass microfiber, Whatman 1PS Phase Separator, and Whatman filter paper Grades 1, 4 and 5 (Table 1). Samples applied to glass fiber papers ionized less efficiently than any of the other papers while the samples applied to the Whatman Grade 1 filter paper were most efficiently ionized (Figure 3). Whatman Grade 5 paper produced 23-27 \% of the ions that were detected from the same samples applied to Whatman Grade 1 paper.
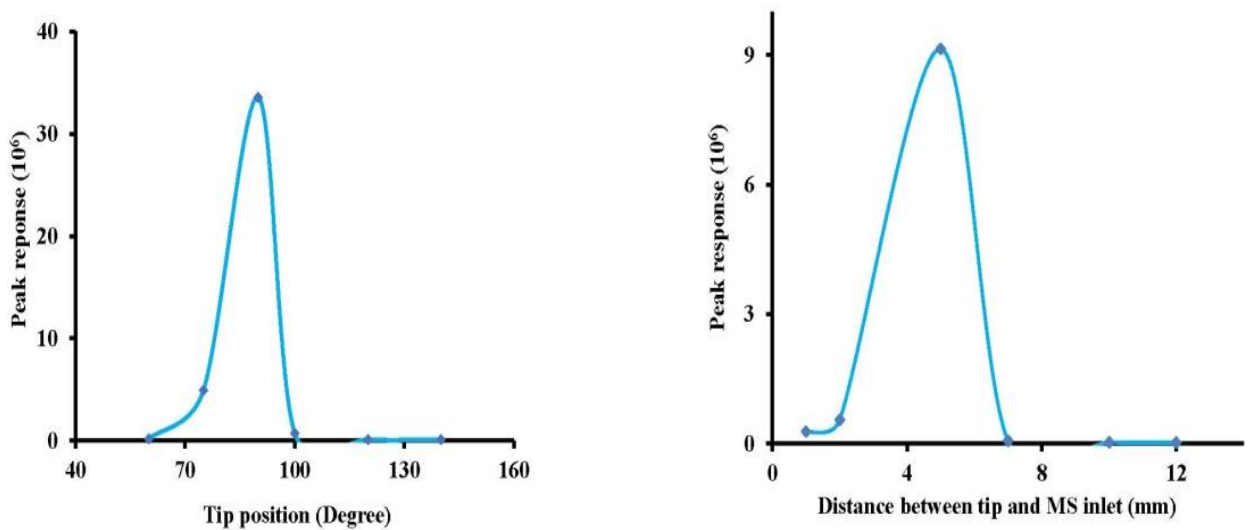

Figure 2. The optimized geometric parameters for paper spray ionization: (A) the distance between the tip of the paper substrate and MS inlet and (B) tip position at angle $60^{\circ}, 75^{\circ}, 90^{\circ}, 120^{\circ}$, and $140^{\circ}$ 


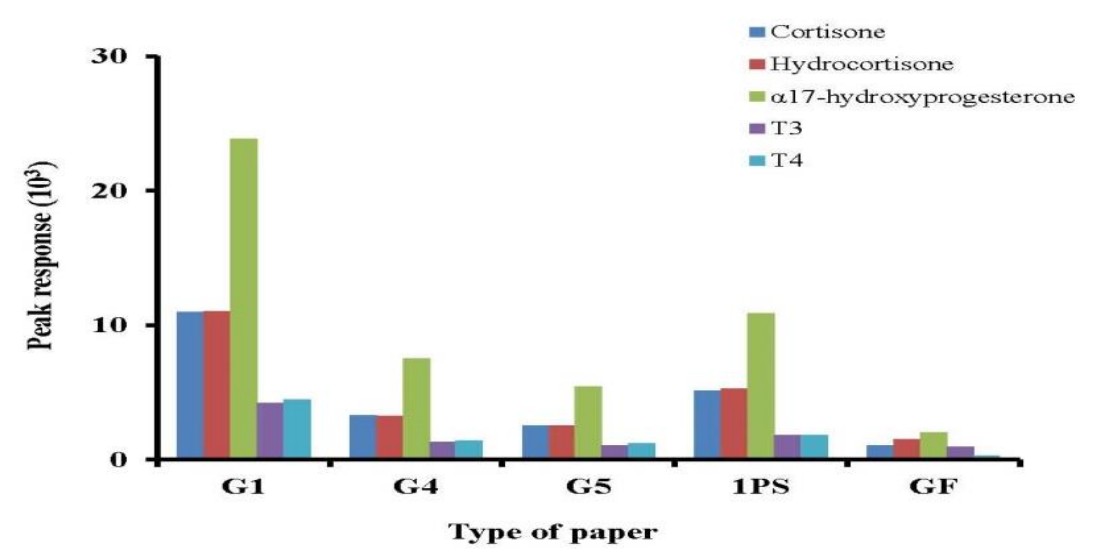

Figure 3. The paper effect (Whatman filter paper grade 1 (G1), grade 4 (G4), grade 5 (G5), glass microfiber filter G6 (GF), and 1PS phase separator (SP) were compared. Whatman filter paper grade 1 showed the highest peak intensity while glass microfiber showed the lowest peak intensity

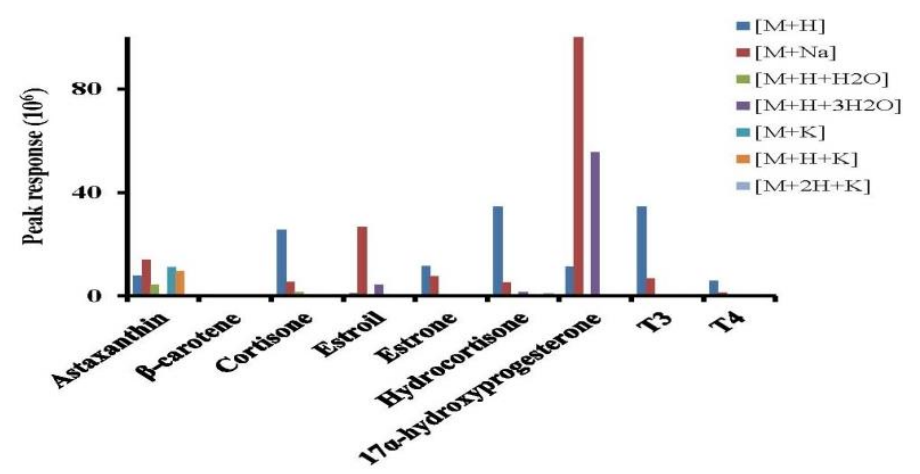

Figure 4. Different adduct peaks formation using Whatman filter paper grade 1 at $10 \mu \mathrm{g} / \mathrm{mL}$ of each analyte

\subsection{Inhibition of Corona Discharge}

To test whether the potential corona discharge affected sample ionization, the custom PSI apparatus was compared to traditional ESI and ESCI. A corona discharge was apparent with the ESI but not the ESCI. Radical cations were detected in the elution of the standard solution from the PSI and the electrospray ionization but not when ESCI was used. Based on these results the presence of corona discharge affects ion formation.

\subsection{Determination of Analytical Performance}

A series of experiments were conducted to determine the sensitivity, selectivity and reproducibility of our custom PSI apparatus using IUPAC figure of merits as dynamic linear range, limit of detection (LOD) and lower limit of quantification (LOQ). The LOD values were found equal to astaxanthin $(28 \mu \mathrm{g} / \mathrm{L}), \beta$-carotene $(30 \mu \mathrm{g} / \mathrm{L})$, caffeine $(20$ $\mu \mathrm{g} / \mathrm{L})$, cortisone $(1.5 \mu \mathrm{g} / \mathrm{L})$, hydrocortisone $(3.8 \mu \mathrm{g} / \mathrm{L}), 17 \alpha$-hydroxyprogesterone $(5 \mu \mathrm{g} / \mathrm{L}), \mathrm{T} 3(25 \mu \mathrm{g} / \mathrm{L}), \mathrm{T} 4(22 \mu \mathrm{g} / \mathrm{L})$. The LOQ values were found equal to astaxanthin $(93.24 \mu \mathrm{g} / \mathrm{L}), \beta$-carotene $(99.9 \mu \mathrm{g} / \mathrm{L})$, caffeine $(66.6 \mu \mathrm{g} / \mathrm{L})$, cortisone $(4.99 \mu \mathrm{g} / \mathrm{L})$, hydrocortisone $(12.65 \mu \mathrm{g} / \mathrm{L}), 17 \alpha$-hydroxyprogesterone $(16.65 \mu \mathrm{g} / \mathrm{L}), \mathrm{T} 3(83.25 \mu \mathrm{g} / \mathrm{L}), \mathrm{T} 4(73.26 \mu \mathrm{g} / \mathrm{L})$ (Table 3). 
Table 3. Analytical performance of selected compounds

\begin{tabular}{lccc}
\hline \multicolumn{1}{c}{ Compounds } & $\mathbf{Y}=\mathbf{m X}+\mathbf{b}$ & $\mathbf{R}^{2}$ & LOQ $(\mu \mathrm{g} / \mathbf{L})$ \\
\hline 17a-hydroxyprogesterone & $\mathrm{Y}=1 \mathrm{e}+06 \mathrm{X}+3 \mathrm{E}+06$ & 0.9906 & 5 \\
\hline Astaxanthin & $\mathrm{Y}=150274 \mathrm{X}+25695$ & 0.9996 & 28 \\
\hline$\beta$-carotene & $\mathrm{Y}=0.0349 \mathrm{X}+0.0058$ & 0.9834 & 30 \\
\hline$\beta$-ionone & $\mathrm{Y}=3217.2 \mathrm{X}+9661.2$ & 0.9973 & 300 \\
\hline Caffeine & $\mathrm{Y}=1344.1 \mathrm{X}+1820$ & 0.9180 & 20 \\
\hline Cortisone & $\mathrm{Y}=1 \mathrm{E}+06 \mathrm{X}+3 \mathrm{E}+06$ & 0.9967 & 1.5 \\
\hline Hydrocortisone & $\mathrm{Y}=304142 \mathrm{X}+270936$ & 0.9904 & 3.8 \\
\hline Isophorone & $\mathrm{Y}=3283.6 \mathrm{X}+10782$ & 0.9941 & 100 \\
\hline Kaempferol & $\mathrm{Y}=280.01 \mathrm{X}+547.36$ & 0.9957 & 290 \\
\hline Quercetin & $\mathrm{Y}=127.71 \mathrm{X}+51.57$ & 0.9931 & 500 \\
\hline Safranal & $\mathrm{Y}=494.52 \mathrm{X}+2754.5$ & 0.9980 & 300 \\
\hline $\mathrm{T}_{3}$ & $\mathrm{Y}=82679 \mathrm{X}+51888$ & 0.9810 & 25 \\
\hline $\mathrm{T}_{4}$ & $\mathrm{Y}=25874 \mathrm{X}+127967$ & 0.9936 & 22 \\
\hline
\end{tabular}

\subsection{Contribution of the Paper to the Sample Matrix}

Elution of the paper with methanol into the TOF-MS revealed that the paper matrix contributes significantly to the total ion counts. The total number of distinct ion signals eluted from the tested paper was over thousand with an average of 23,091 $\pm 2,900$ ion signals. The eluted ion signals were detected per a paper in the absence of samples.

\subsection{Mobilization of Ions From the Paper Surface}

Adduct peaks were observed in standards eluted from the paper surface with the $[\mathrm{M}+\mathrm{Na}]^{+}$as most predominant. For example, the peaks at $\mathrm{m} / \mathrm{z} 619,383,311,385$, and 799 indicated the $[\mathrm{M}+\mathrm{Na}]^{+}$of astaxanthin, cortisone, estriol, hydrocortisone, and T4 respectively. Other adducts peaks such as $[\mathrm{M}+\mathrm{K}]^{+}$were also observed for astaxanthin, $\beta$-carotene, and hydrocortisone, respectively (Figure 4).

\subsection{Interaction of Paper Substrates With Atmospheric Water}

Investigations of the interaction of standard solutions with the paper surface indicated that atmospheric water is required for ionization. Application of standard solutions to oven-dried paper reduced sample detection when non-polar solvents were used. However, standards eluted from ambient Whatman Grade 1 paper with non-polar solvents were detected as $[\mathrm{M}+\mathrm{H} 2 \mathrm{O}]^{+}$and $[\mathrm{M}+\mathrm{H}+(\mathrm{H} 2 \mathrm{O}) 2]^{+}$as well as $[\mathrm{M}+\mathrm{Na}+\mathrm{H} 2 \mathrm{O}]^{+}$and $[\mathrm{M}+\mathrm{K}+\mathrm{H} 2 \mathrm{O}]^{+}$(Figure 4).

\subsection{Comparison of PSI and Two Standard Separation Methods}

In order to estimate the efficiency of PSI for sample component separation, several paper substrates with a range of length $(0.5-5 \mathrm{~cm})$ were prepared. A mixture solution of natural pigments, including astaxanthin, zeaxanthin, and crocin was used for this study. The paper was placed at $5 \mathrm{~mm}$, and $3.5 \mathrm{KV}$ of voltage was applied. The flow has a constant value of $10 \mu \mathrm{L} / \mathrm{min}$ with all lengths of the paper substrate. It was provided that the total time of analysis increased with increasing the length of the substrate. Furthermore, RSD \% was obtained in order to study the precision of this experiment. The values of RSD \% were less than $8 \%$.

In order to determine the capability of paper spray ionization for sample component separation Retention factor (Rf) values of three pigments (astaxanthin, zeaxanthin, and crocin) were obtained. Furthermore, the results were compared in Rf values that obtained by two standard methods, including paper chromatography and thin layer chromatography (Figure 5). Rf values were within a range of $0.80-0.83$ for astaxanthin using the three methods. Rf values were within ranges of 0.77-0.78 and 0.95-0.98 for zeaxanthin and crocin, respectively. The possibility that compounds may be separated on the paper substrate was investigated by comparison to the retention factors (Rf) of astaxanthin, crocin, and zeaxanthin on 
traditional paper chromatography and thin layer chromatography (TLC). Compounds separated in the PSI with Rf values that were consistent to the traditional techniques (Table 4).

Table 4. Comparison between paper spray ionization and two standard methods, including (a) paper chromatography and (b) thin layer chromatography

\begin{tabular}{|c|c|c|c|c|c|}
\hline \multirow{2}{*}{ Length (cm) } & \multirow{2}{*}{$\begin{array}{l}\text { Flow rate } \\
(\mu \mathrm{L} / \mathrm{min} .)\end{array}$} & \multicolumn{3}{|c|}{ Retention Factor ( $R \mathbf{f}$ ) } & \multirow{2}{*}{ RSD $\%$} \\
\hline & & Astaxanthin & Zeaxanthin & Crocin & \\
\hline 0.5 & 10 & - & - & - & 7 \\
\hline 2 & 10 & 0.79 & 0.79 & 0.98 & 6 \\
\hline 4 & 10 & 0.80 & 0.78 & 0.97 & 7 \\
\hline 5 & 10 & 0.81 & 0.77 & 0.95 & 8 \\
\hline $\begin{array}{c}\text { Paper } \\
\text { Chromatography } \\
(5 \mathrm{~cm})\end{array}$ & - & 0.85 & 0.78 & 0.96 & - \\
\hline $\begin{array}{c}\text { Thin Layer } \\
\text { Chromatography } \\
(5 \mathrm{~cm})\end{array}$ & - & 0.83 & 0.77 & 0.95 & - \\
\hline
\end{tabular}
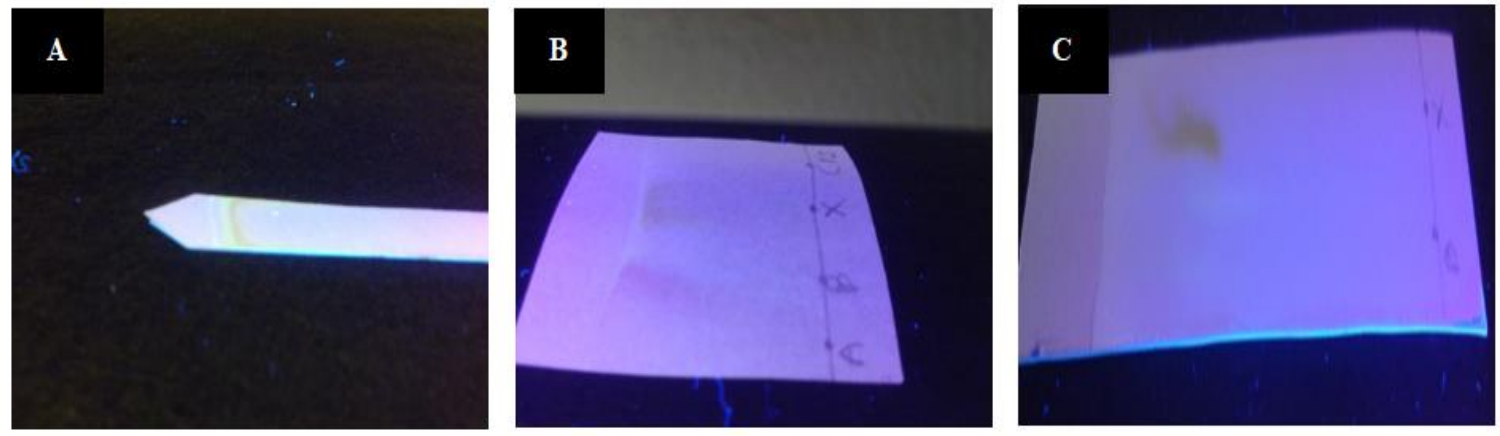

Figure 5. The natural pigments solution (astaxanthin, zeaxanthin, and crocin) that was separated using paper spray ionization (A), paper chromatography (B) and thin layer chromatography (C)

\subsection{Silica Nanoparticles-Paper Spray Ionization Mass Spectrometry}

SEM spectra of paper substrate before and after soaking in the silicon solution show that the nanoparticles are both embedded into the pores and adhering to the fibers (Figure 6). Analysis of the paper found silicon at 30-36\% wt after 72 $\mathrm{h}$ soaking. The silicon nanoparticals significantly increased the elution of the standards from the paper surface. While most standards were detected as the predominant ion $[\mathrm{M}+\mathrm{H}]^{+}$, kaempferol, and quercetin had the radical cation as a predominant ion (Table 5). 
Table 5. Applied voltages and major ions using standard and coated paper

\begin{tabular}{ccccc}
\hline \multirow{2}{*}{ Compound } & \multicolumn{2}{c}{ With $\mathrm{SiO}_{2}$ nanoparticles } & \multicolumn{2}{c}{ Without $\mathrm{SiO}_{2}$ nanoparticles } \\
\cline { 2 - 5 } & $\begin{array}{c}\text { Ion } \\
\text { formation }\end{array}$ & Capillary & Ion formation & Capillary \\
\hline Isophorone & {$[\mathrm{M}+\mathrm{H}]^{+}$} & 3.8 & {$[\mathrm{M}+\mathrm{H}]^{+}$} & 3.3 \\
\hline Safranal & {$[\mathrm{M}+\mathrm{H}]^{+}$} & 3.8 & {$[\mathrm{M}+\mathrm{H}]^{+}$} & 3.3 \\
\hline Kaempferol & {$[\mathrm{M}]^{+}$} & 3.8 & {$[\mathrm{M}+\mathrm{H}]^{+}$} & 3.3 \\
\hline Quercetin & {$[\mathrm{M}]^{+}$} & 4.1 & {$[\mathrm{M}+\mathrm{H}]^{+}$} & 3.5 \\
\hline p-carotene & {$[\mathrm{M}]^{+}$} & 4.1 & {$[\mathrm{M}+\mathrm{H}]^{+}$} & 3.5 \\
\hline Astaxanthin & {$[\mathrm{M}+\mathrm{H}]^{+}$} & 4.1 & {$[\mathrm{M}+\mathrm{H}]^{+}$} & 3.5 \\
\hline $\begin{array}{c}\text { l7a- } \\
\text { hydroxyprogestero } \\
\text { ne }\end{array}$ & {$[\mathrm{M}+\mathrm{H}]^{+}$} & 3.8 & {$[\mathrm{M}+\mathrm{H}]^{+}$} & 3.3 \\
\hline Cortisone & {$[\mathrm{M}+\mathrm{H}]^{+}$} & 4.1 & {$[\mathrm{M}+\mathrm{H}]^{+}$} & 3.5 \\
\hline Hydrocortisone & {$[\mathrm{M}+\mathrm{H}]^{+}$} & 4.1 & {$[\mathrm{M}+\mathrm{H}]^{+}$} & 3.5 \\
\hline T & {$[\mathrm{M}+\mathrm{H}]^{+}$} & 4.1 & {$[\mathrm{M}+\mathrm{H}]^{+}$} & 3.5 \\
\hline
\end{tabular}
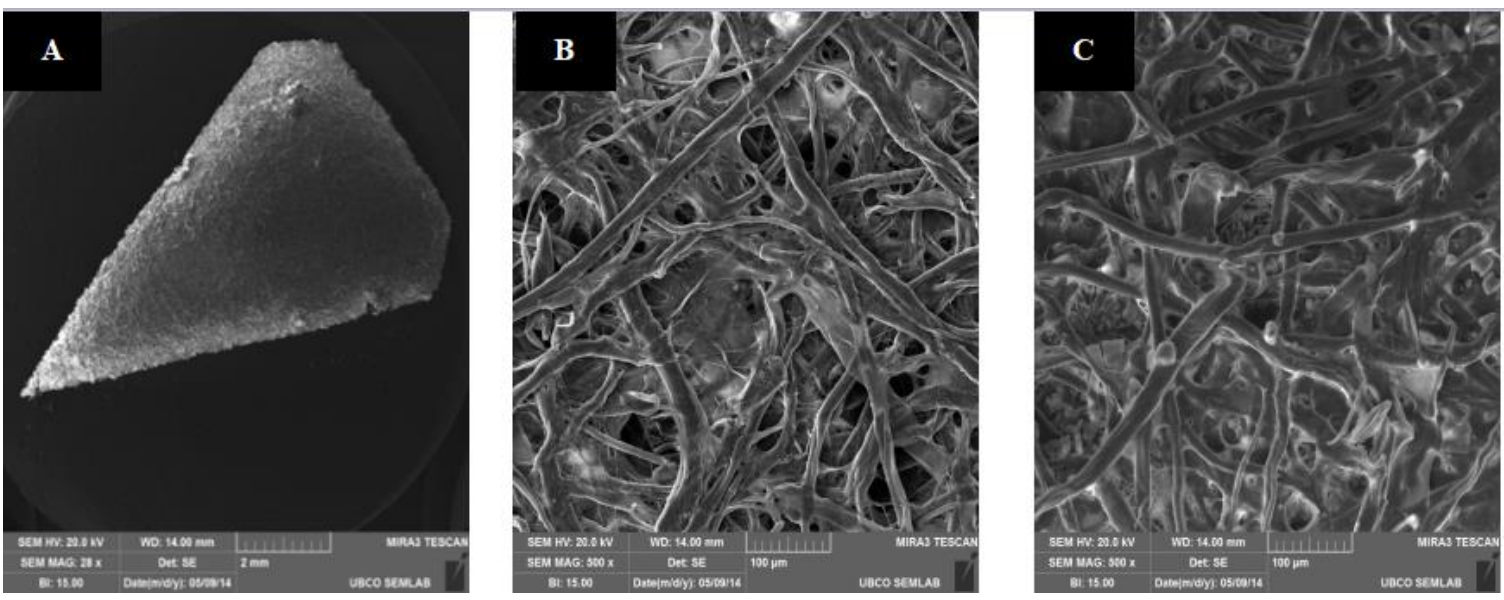

Figure 6. Scanning electron microscopy images of paper substrate (whatman filter paper grade 1) after coated with silica nanoparticles film (A). Scanning electron microscopy analysis spectra of the paper substrate after immersion in silica nanoparticles solution 15 minutes (B) and 72 minutes (C). The spectra showed the existence of silica nanoparticles

\section{Discussion}

New aspects of paper were investigated in this work. Together, the physical properties of the paper substrate were optimized in order to investigate the interactions of the paper with the surrounding environment during analysis, we hypothesized that ionization mechanism and efficiency are affected by several factors, which are discussed as below.

\subsection{Importance of the Corona Discharge}

The corona discharge was seen when the custom PSI apparatus was too close to the inlet cone. Previous researchers have described an electron transfer reaction by corona discharge that neutralizes ions in some methods (Zhang, Cooks, \& 
Ouyang, 2012; Li, Wang, Ouyang, \& Cooks, 2011; Tsao, 2012). The corona discharge is a process by which a current flow from an electrode with a high-applied voltage. It is usually forming at highly sharp section on electrode, such as sharp corners (Zhang et al., 2012, Kebarle, \& Tang, 1993; Liu et al., 2010). The ions generated eventually pass charge to nearby areas of lower potential (MS inlet), or recombine to form neutral molecules. This corona produces when the applied potential is large enough (Zhang et al., 2012, Kebarle, \& Tang, 1993; Liu et al., 2010; Espy et al., 2012; Yang et al., 2012). Thus, the solution at the tip ionizes and it becomes conductive. If a tip of the paper has a sharp point, the electric field strength around that point will be higher than other regions. It was demonstrated that the area near the sharp point can become ionized (partially conductive), while regions more distant do not (Zhang et al., 2012, Kebarle, \& Tang, 1993; Liu et al., 2010; Espy et al., 2012; Yang et al., 2012). At a sufficiently high voltage between the electrodes, the discharge starts at the tip. There are two possible ways to create the corona discharge on the paper tip either as an electric layer on the paper substrate that spreads across the paper at a sufficiently high voltage or a discharge that develops when the voltage difference between the paper substrate and MS inlet cone is high.

\subsection{Atmospheric Water Adsorbed to Paper}

Drying the paper to complete dryness dampened the ion signals that were generated with non-polar eluents. The previous works on PSI/MS discovered that polar compounds were analyzed using a non-polar solvent (Yang et al., 2012) but a mechanism was not proposed. The current results indicate that ambient water within the paper may be mobilized within the solvent/sample matrix and associate with the analytes. Up to three water molecules were detected in association with the analytes indicating that the presence of some water is required. It is likely that residual water molecules are naturally associated the hydroxyl groups of the cellulose paper thereby creating a conductive surface. When voltage is applied, a sustained spray of eluent including eluted water molecules is created. When the paper was dried, the ambient water was evaporated and the applied current was insufficient to induce ionization. The detection of $[\mathrm{M}+\mathrm{Na}]^{+},[\mathrm{M}+\mathrm{K}]+$ and $[\mathrm{M}+\mathrm{K}+\mathrm{H} 2 \mathrm{O}]^{+}$adducts eluted by non-polar solvents is further evidence that the solvent and water molecules moving across the paper substrate solubilize and ionize these ions. Future experiments should quantify the water content in the PSI spray and determine whether ambient water enables electrical current to be conducted across the paper and within the sample fluid when high voltage is applied. These experiments would be quite difficult since removing all ambient water from the mass spectrometer environment is challenging.

\subsection{PSI as Separation/Ionization Technique}

The custom-built PSI apparatus enables the direct introduction of ions into any MS following a classic paper chromatography separation. The paper substrate effectively separated a mixture of carotenoids within short time with high efficiency. Carotenoids are normally analyzed by liquid chromatography requiring large instrumentation (Verma, \& Middha, 2010). The PSI method for separation and detection of these compounds and similar compounds could improve the analysis with short time and might be miniaturized into a portable detection apparatus using a modified PSI for field applications.

\subsection{Porosity of Paper}

In general, the macro molecules required time (approximately 90 seconds) to be extracted from the surface more than the smaller molecules (approximately 30 seconds). It is possible that the difference in retention is a result of the size of pores in the paper since different papers had different capacity for ion production. Silicon nanoparticles have been used as a proton source on the surface for laser desorption ionization, matrix assisted laser desorption/ionization (MALDI), and secondary ion mass spectrometry (Liu, \& He, 2008; Tsao, Yang, Chung, 2012; Gulin, Mochalova, Denisov, \& Nadtochenko, 2014). Protons are generated when the $\mathrm{SiO} 2$ nanoparticles are hydrolyzed in the presence of water or organic solvent to form silicic acid Si (OH)4 (Liu et al., 2008; Gulin et al., 2014). Recently, it was investigated Si nanoparticles on solid substrates as a mechanism for improved ionization but this approach has not been tried with PSI (Tsao et al., 2012). Silica nanoparticles formed an association with the cellulose in the paper that included both filling pores and adhering to the fibers and appear to be evenly spread across the surface of the paper. This interaction between silica nanoparticles and cellulose molecules may include hydrogen bonding to the surface oxygen (Tsao et al., 2012). Analysis of standards indicated that the ionization efficiency was increased but the mechanism was not fully elucidated.

In order to study the effect of silica nanoparticles film on the ionization efficiency, peak responses were compared for various analytes.

The proposed method was compared with the standard method (absent of silica nanoparticles film) in order to investigate the effect of the film. Applied potential and flow rate were optimized for each method. A wide range of 1.5-4.5 KV was used in order to optimize the applied potential. According to the present work results a range of 3.8-4.1 KV were found to be optimal with the present of silica nanoparticles film while it was within range of 3.3-3.5 KV without the film. The difference in the applied potential with and without the film was only $0.5 \mathrm{KV}$. 
PSI mass spectra were also compared with and without the silica nanoparticles. The results show that the protonated peaks were observed for all tested compounds using the standard paper and the paper coated with the film except for three compounds, kaempferol, quercetin and $\beta$-carotene. These compounds gave a radical cation as a predominant ion. In addition, the variety in the peak responses was observed using two solvents. Mixtures of methanol/water and dichloromethane/isopropanol solvents $(9: 1, \mathrm{v} / \mathrm{v})$ were used in this study (Figure 7).
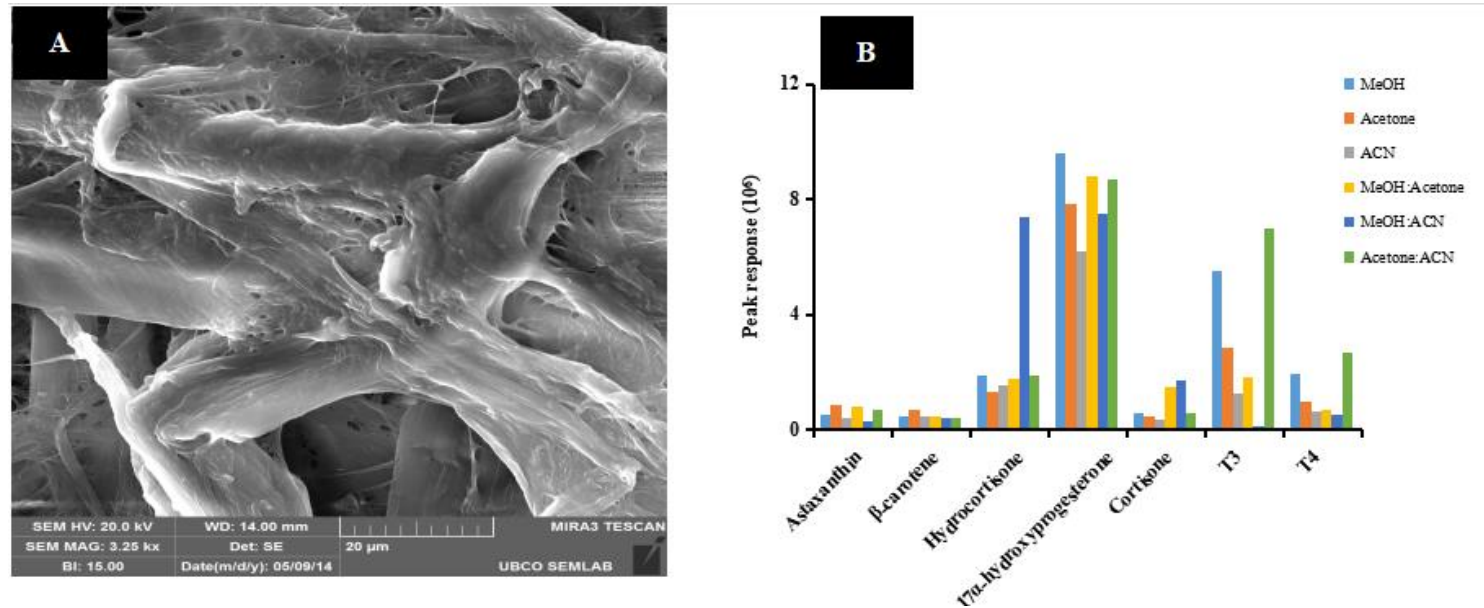

Figure 7. Scanning electron microscope image of the analyte that adsorbed on the paper substrate (thin fiber) where it was not obtained on the paper substrate without the addition of the analyte (A), and the optimal solvent for each compound, where methanol and acetone was obtained as optimal solvent for progesterone and carotenoids (B). A mixture of acetone and acetonitrile $(\mathrm{ACN})$ was the optimal solvent for thyroid hormones. For steroid hormones, the optimal solvent was a mixture of methanol and acetonitrile

The results showed peak responses with the coated paper higher than the standard paper for various compounds, including isophorone, safranal, $\beta$-ionone, kaempferol, astaxanthin, 17 $\alpha$-hydroxyprogesterone, cortisone, hydrocortisone, and T4 (Figure 8). Based on this result, either the concentration of proton increased or the analytes were less strongly bound to the paper because the nanoparticles filled the pores in the paper. Silica nanoparticles boost the ionization efficiency on the paper. This result is in agreement with a previous work where the researchers observed increasing in the peak response of protein with silica nanoparticles using secondary ion mass spectrometry (Omanovic-Miklicanin et al., 2014). Further addition, the recovery values were calculated for several analytes in order to determine the accuracy of the developed method. The values were obtained with pure solution of the analyte using coated paper. The results revealed recovery values in the presence of silica nanoparticles film reached to $100 \%$. Based on this result, the coated paper has a good accuracy.

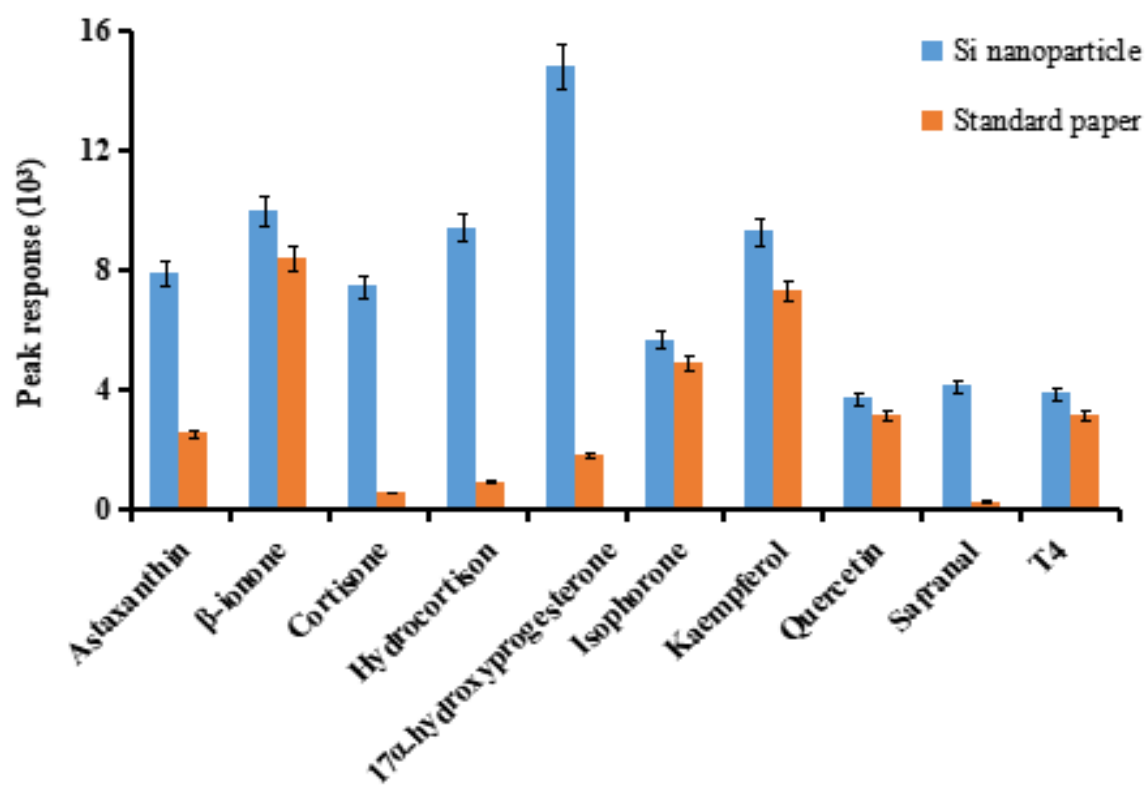

Figure 8. Comparison of peak responses with standard and coated papers. The plot show peak responses with coated paper higher than standard paper 
It was indicated that $\mathrm{Si} /$ cellulose interaction (hybrid) is an important factor that affects the silica nanoparticles film formation on the substrate. Poor interaction leads to variation in the ionization efficiency (Tsao et al., 2012). In order to produce an efficient $\mathrm{Si} /$ cellulose hybrid on the paper, the nanoparticles must be strongly adsorbed on the paper whilst avoiding damage of the paper. To study the distribution of nanoparticles on the paper four papers were immersed in the silica nanoparticles solution for several days. The SEM images showed that $\mathrm{SiO} 2$ nanoparticles spread over the papers with high efficiency with increasing the time of immersion of the paper substrate in the silica nanoparticles solution. At short time (15 minutes), the silicon ranged between 2.2-36\% wt., where not all pores were filled by silica nanoparticles. For longer time, the silicon ranged between 30-36 \% wt. Most pores were filled with the nanoparticles. Based on these results, the paper substrates were immersed in the solution for $72 \mathrm{~h}$, and used for the rest experiments. However, the formation of an over oxidized silicon nanoparticles layer (after $72 \mathrm{~h}$ ) slows down overall peak intensity for some compounds in the current study. The aggregation state of nanoparticles might affect the ionization efficiency. Therefore, the degree of the silica nanoparticles distribution on the paper needs to be carefully monitored to achieve optimal MS results.

The recovery percent of the proposed method was obtained at three levels using saffron samples. The results show that the recovery percent was improved and lay within range of 90-98\% in the present of silica nanoparticles film while it was lay within range of $80-99 \%$ without the film.

\section{Conclusion}

A custom-built apparatus was developed that supported a paper substrate for PSI of a diverse range of chemical standards. Ionization efficiency depended on the position, the type of paper, the application of solvent and various other factors that must be optimized before such devices can be used. Studies of the degradation of paper demonstrated that the paper surface is not inert and paper-compounds can be introduced into the sample matrix thereby complicating the analysis. The PSI source can act as a separation technique for complex samples and could be adapted to many different types of analysis in addition to mass spectrometry. The observation that ions were generated in the presence of a non-polar eluent led to the discovery that ambient water on the paper surface is crucial for PSI, Silica nanoparticles acted as an adjuvant to the paper and provided protons for increased ionization efficiency. Together, these data increase our understanding of the role of paper substrates in chemical analyses and form the foundation for future work to develop novel analytical tools using paper as a method for introduction of samples.

\section{Notes}

The author declares no conflicts of interest.

\section{Acknowledgment}

The author would like to thank Western Economic Diversion and BCKDF for funding to purchase instrumentation. Our thanks to Czech Republic, SEMLAB facilities and the Charles E. Fipke Foundation in UBCO- Canada. Our grateful acknowledgement goes to King Abdulaziz University, Kingdom of Saudi Arabia for the financial support.

\section{References}

Bills, J. B., \& Manicke, E. N. (2016). Development of a prototype blood fractionation cartridge for plasma analysis by paper spray mass spectrometry, Clinical Mass Spectrometry, 2, 18-24. https://doi.org/10.1016/j.clinms.2016.12.002

Carvalho, T. C. D., Tosato, F., Souza, L. M., Santos, H., Merlo, B. B., Ortiz, R. S.,... Vaz, B. G. (2016). Thin layer chromatography coupled to paper spray ionization mass spectrometry for cocaine and its adulterants analysis. Forensic Science International, 262, 56-65. https://doi.org/10.1016/j.forsciint.2016.02.039

Espy, R., Muliadi, A., Ouyang, Z., \& Cooks, R. (2012). Spray Mechanism in Paper Spray Ionization. International Journal of Mass Spectrometry, 167-171. https://doi.org/10.1016/j.ijms.2012.06.017

Gulin, A., Mochalova, M., Denisov, N., \& Nadtochenko, V. (2014). Secondary Ions Mass Spectrometry Signal Enhancement of Phosphatidylcholine Dioleoyl on Enlarged Nanoparticles Surface, Applied Surface Science, 316, 36-41. https://doi.org/10.1016/j.apsusc.2014.07.102

Kebarle, P., \& Tang, L. (1993). From Ions in Solution to Ions in the Gas Phase-The Mechanism of Electrospray Mass Spectrometry. Analytical Chemistry, 65, 972-986. https://doi.org/10.1021/ac00070a001

Kim, D., Ha, S. Y., An, J. G., Cha, S., Yim, U. H., \& Kim, S. (2018). Estimating degree of degradation of spilled oils based on relative abundance of aromatic compounds observed by paper spray ionization mass spectrometry. Journal of Hazardous Materials, 359, 421-428. https://doi.org/10.1016/j.jhazmat.2018.07.060

Li, A., Wang, H., Ouyang, Z., \& Cooks, R. (2011). Paper Spray Ionization of Polar Analytes Using Non-Polar Solvents. Chemical Communication, 47, 2811-2813. https://doi.org/10.1039/c0cc05513a 
Liu, J., He, Y., Chen, S., Ma, M., Yao, S., \& Chen, B. (2017). New urea-modified paper substrate for enhanced analytical performance of negative ion mode paper spray mass spectrometry. Talanta, 166, 306-314. https://doi.org/10.1016/j.talanta.2017.01.076

Liu, J., Wang, H., Manicke, N., Lin, J., Cooks, R., \& Ouyang, Z. (2010). Development, Characterization, and Application of Paper Spray Ionization. Analytical Chemistry, 82, 2463-2471. https://doi.org/10.1021/ac902854g

Liu, Q., \& He, L. (2008). Quantitative Study of Solvent and Surface Effects on Analyte Ionization in Desorption Ionization on Silicon (DIOS) Mass Spectrometry. Journal of the American Society for Mass Spectrometry, 19, 8-13. https://doi.org/10.1016/j.jasms.2007.10.002

Miller, J., \& Miller, N. (1994). Statistics for Analytical Chemistry (4th ed.). New York: NY Ellis Horwood.

Page, J. S., Kelly, R. T., Tang, K., \& Smith, R. D. (2007). Ionization and Transmission Efficiency in an Electrospray Ionization-Mass Spectrometry Interface. Journal of the American Society for Mass Spectrometry, 18, 1582-1590. https://doi.org/10.1016/j.jasms.2007.05.018

Tsao, C., Yang, Z., \& Chung, C. (2012). Preparation of Nanostructured Silicon Surface for Mass Spectrometry Analysis by an All-Wet Fabrication Process Using Electroless Metal Deposition and Metal Assisted Etching. International Journal of Mass Spectrometry, 8-13. https://doi.org/10.1016/j.ijms.2012.05.007

Verma, R. S., \& Middha, D. (2010). Analysis of Saffron (Crocus sativus L.Stigma) Components by LC-MS-MS. Chromatographia, 71, 117-123. https://doi.org/10.1365/s10337-009-1398-Z

Wang, H., Liu, J., Cooks, R., \& Ouyang, Z. (2010). Paper Spray for Direct Analysis of Complex Mixtures Using Mass Spectrometry. Angewandte Chemie, 49, 877-880. https://doi.org/10.1002/anie.200906314

Yang, Q., Wang, H., Maas, J., Chappell, W., Manick, N., Cooks, R., \& Ouyanga, Z. (2012). Paper Spray Ionization Devices for Direct, Biomedical Analysis Using Mass Spectrometry, International Journal of Mass Spectrometry, 312, 201-207. https://doi.org/10.1016/j.ijms.2011.05.013

Yu, M., Wen, R., Jiang, L., Huang, S., Fang, Z., Chen, B, \& Wang, L. (2018). Rapid analysis of benzoic acid and vitamin $\mathrm{C}$ in beverages by paper spray mass spectrometry. Food Chemistry, 268, 411-415. https://doi.org/10.1016/j.foodchem.2018.06.103

Zhang, Z., Cook, R., \& Ouyang, Z. (2012). Paper Spray: A Simple and Efficient Means of Analysis of Different Contaminants in Foodstuffs. Analyst, 137, 2556-2558. https://doi.org/10.1039/c2an35196j

\section{Copyrights}

Copyright for this article is retained by the author(s), with first publication rights granted to the journal.

This is an open-access article distributed under the terms and conditions of the Creative Commons Attribution license (http://creativecommons.org/licenses/by/4.0/). 